\title{
Influenza vaccine update, 2021-22
}

\author{
Give the vaccine by the end of October. The CDC states \\ that COVID-19 vaccines can be co-administered with \\ influenza vaccines, but reactogenicity is possible.
}

$\mathrm{D}$ uring the 2020-2021 influenza season, fewer cases of influenza were reported than in any previous year since 1997, when data were first recorded. ${ }^{1}$ FIGURE $1^{2}$ shows the dramatic decline in the number of influenza-positive clinical samples reported to the Centers for Disease Control and Prevention (CDC) during the 2020-2021 influenza season compared with the 2019-2020 season. There was only one pediatric death attributed to influenza in 2020-2021, compared with a mean of 177 per year in the previous 3 seasons.

Deaths attributed to pneumonia and influenza were recorded over a recent 5-year period, with COVID-19 added in early mid2020 (FIGURE 2). ${ }^{1}$ Total cumulative deaths for 2020-2021 were extremely high, mostly due to COVID-19. Of the relatively few influenza cases last season, $37.5 \%$ were caused by influenza A and $62.5 \%$ by influenza B. The extremely low incidence of influenza precludes determining influenza vaccine effectiveness for last season. ${ }^{1}$

In addition, other common respiratory pathogens-parainfluenza, adenoviruses, rhinoviruses, enteroviruses, and common coronaviruses-circulated last winter at historic lows. ${ }^{3}$ All of these historic lows can be attributed to the measures taken to mitigate the effect of the COVID-19 pandemic, including masks, social distancing, closure of certain venues that normally attract large crowds, and the closure of schools with a resulting increase in schooling at home. With the anticipated relaxation of these measures in 2021-2022, we can expect more influenza and other respiratory ailments due to common pathogens.

\section{Updates to influenza}

vaccine recommendations

At its June 2021 meeting, the Advisory Committee on Immunization Practices (ACIP) approved the influenza vaccine recommendations for the 2021-2022 season. ${ }^{4}$ The central recommendation is unchanged: Everyone $\geq 6$ months of age should receive a vaccine unless they have a contraindication. Updates to the previous recommendations include the content of the 2021 vaccines, the specific vaccines that will be available for different age groups, the timing of vaccine administration, advice on co-administration with COVID-19 vaccines, and the list of contraindications and precautions based on vaccine type. ${ }^{4}$

\section{Viral composition of US vaccines for the 2021-22 season}

The antigens that will be included in the 2021-2022 influenza vaccines are listed in TABLE $1{ }^{4}$ The $B$ strains are the same as last year; the A strains have been updated. The H3N2 strain is the same in all vaccines, but the H1N1 strain differs based on whether the vaccine is egg based or non-egg based. The advantage of non-egg-based vaccines is that the production process does not take as long and can be delayed in an attempt to better reflect the influenza stains in worldwide circulation.

\section{The influenza vaccines expected} to be available for the 2021-22 season

TABLE $2^{4}$ lists the influenza vaccines approved for use in the United States and the ages for which they are approved. ${ }^{4}$ All products for 2021-2022 will be quadrivalent, containing 2 type-A and 2 type-B antigens. The only
Doug Campos-Outcalt, MD, MPA

University of Arizona, Phoenix

Ddougco@email.arizona. edu

The author reported no potential conflict of interest relevant to this article.

doi: 10.12788/jp.0277 
FIGURE 1

Far fewer influenza-positive tests were reported in the 2020-21 season than during the 2019-20 season ${ }^{2}$

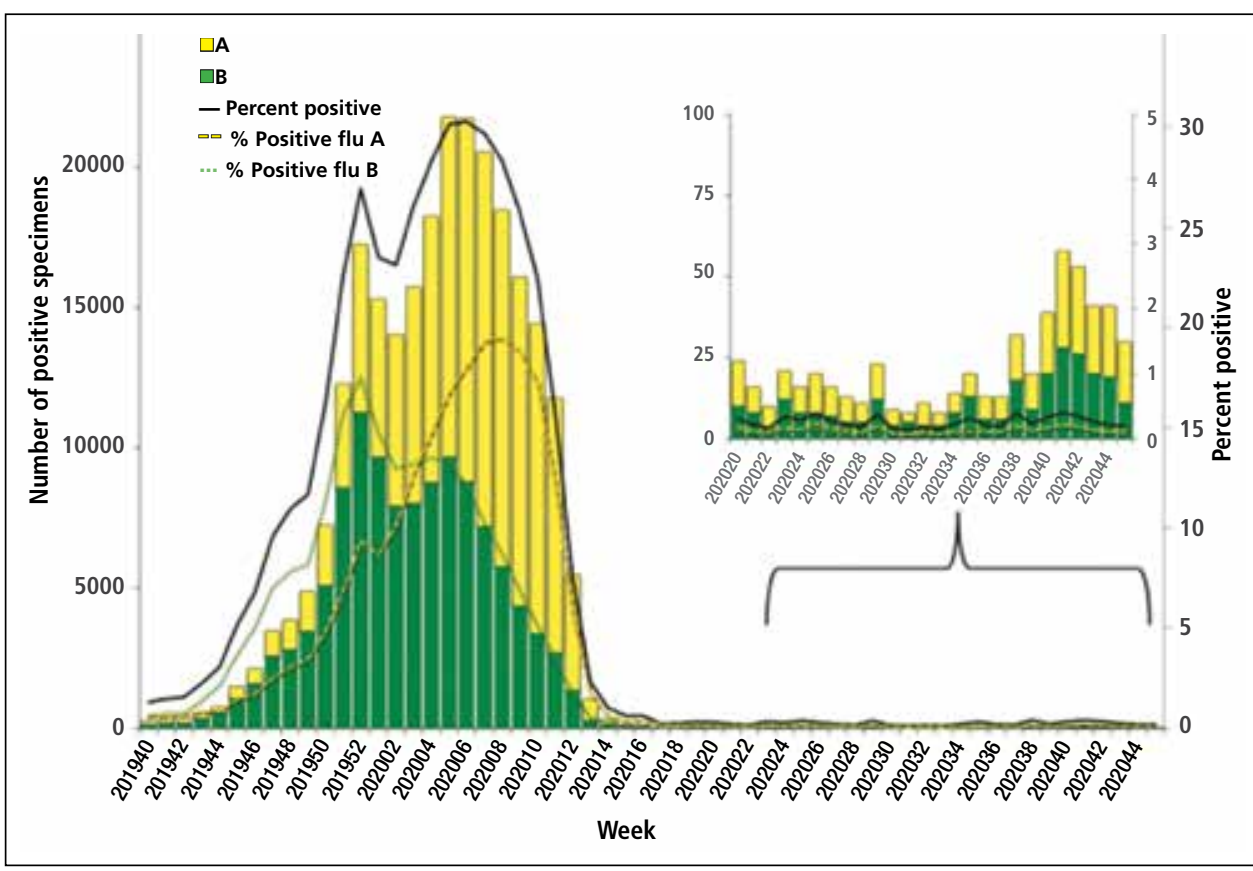

FIGURE 2

\section{US mortality rates for COVID-19 have far exceeded those for pneumonia and influenza ${ }^{1}$}

Five-year data reported through week ending July 24, 2021

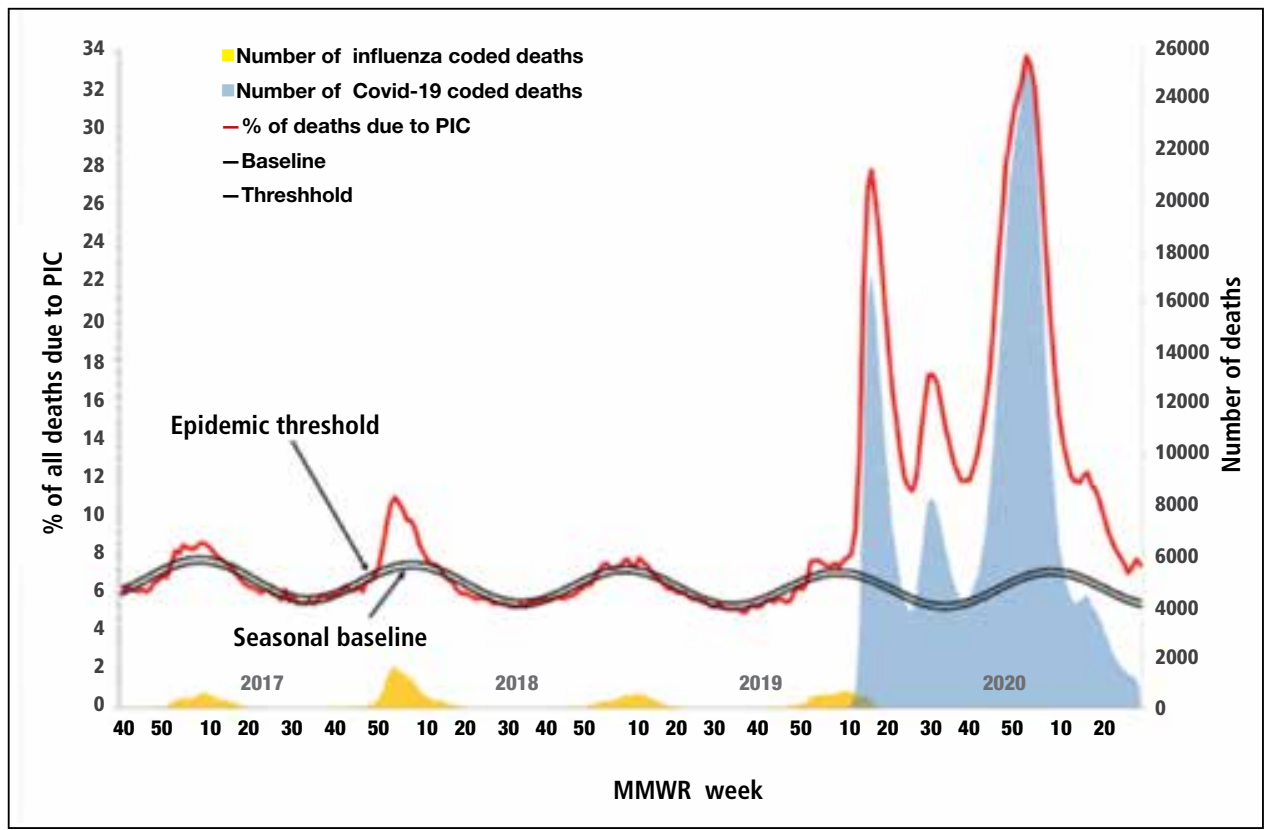

PIC, pneumonia, influenza, and/or COVID-19. change in age indications is that cell culture-based inactivated influenza vaccine (ccIIV4) (Flucelvax Quadrivalent) is now approved for use starting at age 2 years; previously it was approved starting at age 4 years. ${ }^{4}$

\section{Timing of vaccination}

The onsets and peaks of influenza disease occur at different times each year and can also vary by geographic location. An analysis of 36 influenza seasons starting in 1982 showed that peak activity occurred most frequently in February (15 seasons), followed by December (7 seasons), and January and March (6 seasons each). ${ }^{5}$ Only once did peak activity occur in October and once in November. This information, along with observational studies showing the waning of influenza vaccine effectiveness after 5 to 6 months, especially in the elderly, informed the ACIP decision to modify their recommendation on the timing of vaccination. The recommendation now states that vaccine should be administered by the end of October and that July and August would have been too early, especially for older adults.

Children ages 6 months through 8 years who have not been vaccinated previously require 2 doses separated by at least 4 weeks, and the first dose should be administered early enough to allow for the second by the 
end of October. ${ }^{4}$ Children who require only 1 dose can also receive the vaccine as soon as it is available, as there is less evidence that vaccine effectiveness wanes in children.

Earlier administration is also recommended for pregnant women in their third trimester. Delaying vaccination in this group could result in postpartum administration of the vaccine, thereby depriving infants of protection against influenza illness during their first 6 months after birth. ${ }^{4}$

\section{Co-administration of influenza and COVID-19 vaccines}

Current guidance from the CDC states that COVID-19 vaccines can be co-administered with other vaccines including influenza vaccines. ${ }^{6}$ However, there are no data by which to judge the efficacy of each vaccine in coadministration or the potential for increased adverse reactions. ACIP advises caution on 2 points: (1) physicians should watch for updated guidance as more information becomes available, and (2) there is the potential for increased reactogenicity after co-administration, especially with the more reactogenic influenza vaccines: adjuvanted inactivated influenza vaccine (aIIV4) and high-dose inactivated influenza
TABLE 1

\section{1-2022 influenza vaccine composition ${ }^{4}$}

Egg-based IIV4s and LAIV4

A/Victoria/2570/2019 (H1N1)pdm09-like virus ${ }^{\mathrm{a}}$

A/Cambodia/e0826360/2020 (H3N2)-like virus ${ }^{a}$

B/Washington/02/2019 (Victoria lineage)-like virus

B/Phuket/3073/2013 (Yamagata lineage)-like virus

Cell-culture-based IIV4 and RIV4

A/Wisconsin/588/2019 (H1N1)pdm09-like virus ${ }^{a}$

A/Cambodia/e0826360/2020 (H3N2)-like virus ${ }^{a}$

B/Washington/02/2019 (Victoria lineage)-like virus

B/Phuket/3073/2013 (Yamagata lineage)-like virus

IIV4, quadrivalent inactivated influenza vaccine; LAIV4, quadrivalent live-attenuated influenza vaccine; RIV4, quadrivalent recombinant influenza vaccine.

a Updated from 2020-2021 vaccine.

vaccine (HD-IIV4). Moreover, these vaccines and the co-administered COVID-19 vaccine should be injected into different limbs.

\section{Contraindications and precautions}

Serious allergic reactions to influenza vaccines are rare-about 1.3 incidents per million doses administered. ${ }^{7}$ However, a previous severe allergic reaction to a particular vaccine or to any component of the vaccine is a con-

TABLE 2

Influenza vaccines expected to be available in the United States for the 2021-2022 flu season ${ }^{4}$

All vaccines listed are quadrivalent.

\begin{tabular}{|c|c|c|c|c|c|c|}
\hline Vaccine type & $0-6 \mathrm{mo}$ & $6-23 \mathrm{mo}$ & $2-17 y$ & $18-49$ y & $50-64 y$ & $\geq 65 y$ \\
\hline \multirow{4}{*}{$\begin{array}{l}\text { Standard-dose, unadjuvanted } \\
\text { inactivated (IIV4) }\end{array}$} & \multirow{4}{*}{ N/A } & \multicolumn{5}{|c|}{ Afluria Quadrivalent } \\
\hline & & \multicolumn{5}{|c|}{ Fluarix Quadrivalent } \\
\hline & & \multicolumn{5}{|c|}{ FluLaval Quadrivalent } \\
\hline & & \multicolumn{5}{|c|}{ Fluzone Quadrivalent } \\
\hline $\begin{array}{l}\text { Cell culture-based inactivated } \\
\text { (IIV4) }\end{array}$ & \multicolumn{2}{|c|}{ N/A } & \multicolumn{4}{|c|}{ Flucelvax Quadrivalent } \\
\hline Adjuvanted inactivated (allV4) & \multicolumn{5}{|c|}{ N/A } & $\begin{array}{l}\text { Fluad } \\
\text { Quadrivalent }\end{array}$ \\
\hline $\begin{array}{l}\text { High-dose inactivated } \\
\text { (HD-IIV4) }\end{array}$ & \multicolumn{5}{|c|}{ N/A } & $\begin{array}{l}\text { Fluzone } \\
\text { High-dose } \\
\text { Quadrivalent }\end{array}$ \\
\hline Recombinant (RIV4) & \multicolumn{3}{|c|}{ N/A } & \multicolumn{3}{|c|}{ Flublok Quadrivalent } \\
\hline Live attenuated (LAIV4) & \multicolumn{2}{|c|}{ N/A } & \multicolumn{2}{|c|}{ FluMist Quadrivalent } & \multicolumn{2}{|r|}{ N/A } \\
\hline
\end{tabular}

Egg based Non-egg based

IIV4, quadrivalent inactivated influenza vaccine; LAIV4, quadrivalent live attenuated influenza vaccine; N/A, not approved for age group; RIV4, quadrivalent recombinant influenza vaccine. 
traindication for use of that vaccine. In addition, a history of severe allergic reaction to any influenza vaccine is a contraindication for all egg-based vaccines.

There are 2 precautions for all influenza vaccines: a concurrent moderate or severe acute illness (with or without fever), and a history of Guillain-Barré syndrome within 6 weeks of receiving any influenza vaccine. An additional precaution for ccIIV4 and recombinant influenza vaccine (RIV4) is a history of severe allergic reaction after administration of any other influenza vaccine. Administration of RIV4 or ccIIV4 to someone with such a history should occur in a medical setting and be supervised by someone who can recognize and treat a severe reaction.

Live attenuated influenza vaccine (LAIV) continues to have a considerably longer list of contraindications, which can be found in the published recommendations for 2021-2022. ${ }^{8}$

\section{Final advice}

The upcoming influenza season has the potential to be clinically challenging with the possibility of co-existing high rates of both COVID-19 and influenza. Recommend both influenza and COVID-19 vaccination to patients. Also, be sure to encourage and practice other preventive measures such as masking in crowds, frequent hand washing, isolation when sick, respiratory hygiene, and (for physicians) selected prescribing of influenza antiviral medications and meticulous office-based infection control practices. ${ }^{9}$

\section{References}

1. CDC. Weekly U.S. influenza surveillance report. Accessed September 23, 2021. www.cdc.gov/flu/weekly/index.htm

2. CDC. Weekly archives. Accessed September 23, 2021. www.cdc. gov/flu/weekly/weeklyarchives2020-2021/WhoNPHL45.html

3. Olsen SJ, Winn AK, Budd AP, et al. Changes in influenza and other respiratory virus activity during the COVID-19 pandemic - United States, 2020-2021. MMWR Morb Mortal Wkly Rep. 2021;70:1013-1019.

4. Grohskopf L. WG considerations and proposed influenza vaccine recommendations, 2021-22. Presented at the June 24, 2021, meeting of the Advisory Committee on Immunization Practices. Accessed September 23, 2021. www.cdc.gov/vaccines/ acip/meetings/downloads/slides-2021-06/03-influenzagrohskopf-508.pdf

5. CDC. The flu season. Accessed September 23, 2021. www.cdc. gov/flu/about/season/flu-season.htm

6. CDC. Interim clinical considerations for use of COVID-19 vaccines currently approved or authorized in the United States. Accessed September 23, 2021. www.cdc.gov/vaccines/covid-19/ clinical-considerations/covid-19-vaccines-us.html?CDC AA_refVal=https $\% 3 \mathrm{~A} \% 2 \mathrm{~F} \% 2 \mathrm{Fwww}$.cdc.gov\%2Fvaccines\%2F covid-19\%2Finfo-by-product\%2Fclinical-considerations.html \#Coadministration

7. McNeil MM, Weintraub ES, Duffy J, et al. Risk of anaphylaxis after vaccination in children and adults. J Allergy Clin Immunol. 2016;137:868-878.

8. Grohskopf LA, Alyanak E, Ferdinands JM, et al. Prevention and control of seasonal influenza with vaccines: recommendations of the Advisory Committee on Immunization Practices, United States, 2021-22 influenza season. MMWR Morb Mortal Wkly Rep. 2021;70:1-28.

9. CDC. Prevent flu. Accessed September 23, 2021. www.cdc.gov/ flu/prevent/index.html

\section{SHOULDER DISLOCATION}

CONTINUED FROM PAGE 375

8. Lizzio VA, Meta F, Fidai M, et al. Clinical evaluation and physical exam findings in patients with anterior shoulder instability. Curr Rev Musculoskelet Med. 2017;10:434-441. doi: 10.1007/ s12178-017-9434-3

9. Farber AJ, Castillo R, Clough M, et al. Clinical assessment of three common tests for traumatic anterior shoulder instability. J Bone Joint Surg Am. 2006;88:1467-1474. doi: 10.2106/JBJS.E.00594

10. Robinson CM, Shur N, Sharpe T, et al. Injuries associated with traumatic anterior glenohumeral dislocations. J Bone Joint Surg Am. 2012;94:18-26. doi: 10.2106/JBJS.J.01795

11. de Laat EA, Visser CP, Coene LN, et al. Nerve lesions in primary shoulder dislocations and humeral neck fractures. A prospective clinical and EMG study. J Bone Joint Surg Br. 1994;76: 381-383.

12. Avis D, Power D. Axillary nerve injury associated with glenohumeral dislocation: a review and algorithm for management. EFORT Open Rev. 2018;3:70-77. doi: 10.1302/2058-5241.3.170003

13. Drury JK, Scullion JE. Vascular complications of anterior dislocation of the shoulder. Br J Surg. 1980;67:579-581. doi: 10.1002/ bjs. 1800670817

14. Lafuente JLA, Marco SM, Pequerul JMG. Controversies in the management of the first time shoulder dislocation. Open Orthop J. 2017;11:1001-1010. doi: 10.2174/1874325001711011001

15. te Slaa RL, Brand R, Marti RK. A prospective arthroscopic study of acute first-time anterior shoulder dislocation in the young: a five-year follow-up study. J Shoulder Elbow Surg. 2003;12:5 29-534. doi: 10.1016/s1058-2746(03)00218-0

16. Kavaja L, Lähdeoja T, Malmivaara A, et al. Treatment after traumatic shoulder dislocation: a systematic review with a network meta-analysis. Br J Sports Med. 2018;52:1498-1506. doi: 10.1136/ bjsports-2017-098539

17. Krych AJ, Sousa PL, King AH, et al. The effect of cartilage injury after arthroscopic stabilization for shoulder instability. Orthopedics. 2015;38:e965-e969. doi: 10.3928/01477447-20151020-03

18. Polyzois I, Dattani R, Gupta R, et al. Traumatic first time shoulder dislocation: surgery vs non-operative treatment. Arch Bone Jt Surg. 2016;4:104-108.

19. Maman E, Harris C, White L, et al. Outcome of nonoperative treatment of symptomatic rotator cuff tears monitored by magnetic resonance imaging. J Bone Joint Surg Am. 2009;91:1898-1906. doi: 10.2106/JBJS.G.01335

20. Safran O, Schroeder J, Bloom R, et al. Natural history of nonoperatively treated symptomatic rotator cuff tears in patients 60 years old or younger. Am J Sports Med. 2011;39:710-714. doi $10.1177 / 0363546510393944$

21. Parnes N, Bartoszewski NR, Defranco MJ. Arthroscopic repair of full-thickness rotator cuff tears in active patients younger than 40 years: 2- to 5-year clinical outcomes. Orthopedics 2018;41 e52-e57. doi: 10.3928/01477447-20171114-02

22. Sofu H, Gürsu S, Koçkara N, et al. Recurrent anterior shoulder instability: review of the literature and current concepts. World J Clin Cases. 2014;2:676-682. doi: 10.12998/wjcc.v2.i11.676

23. Emond M, Le Sage N, Lavoie A, et al. Clinical factors predicting fractures associated with an anterior shoulder dislocation. Acad Emerg Med. 2004;11:853-858. doi: 10.1111/j.1553-2712.2004. tb00768.x

24. Parnes N, Jupiter JB. Fixed-angle locking plating of displaced proximal humerus fractures. Instr Course Lect. 2010;59:539-552. 